\title{
Volatility Effect on the Adoption and Valuation of Tokenomics
}

\author{
Jollen Chen \\ The Flowchain Foundation \\ Singapore \\ jollen@flowchain.io
}

\author{
Dung-Cheng Lin \\ Department of Mathematics, \\ National Taiwan University \\ Taipei, Taiwan \\ dungchenglin@ntu.edu.tw
}

\author{
Chuan-Hsiang Han* \\ Department of Quantitative Finance, \\ National Tsing-Hua University \\ Hsinchu, Taiwan \\ chhan@mx.nthu.edu.tw
}

\begin{abstract}
Motivated by financial analytics for volatile behavior of cryptoassets such as Bitcoin and Ether, we generalize a recent paper of Cong, Li and Wang (2019) to take time-varying volatility process into account and investigate its effect on tokenomics including user adoption and price valuation. Our finding reveals that high adoption of userbase and stability of token's price are associated with volatility reduction of the platform productivity. In addition, this model can be modified for IoT blockchain by considering IoT nodes as the user in a peer-to-peer network.
\end{abstract}

\section{CCS CONCEPTS}

- Mathematics of computing $\rightarrow$ Stochastic processes; • Theory of computation $\rightarrow$ Random walks and Markov chains; • Computing methodologies $\rightarrow$ Model development and analysis;

\section{KEYWORDS}

tokenomics, volatility, userbase adoption, price valuation, platform productivity, IoT blockchain

\section{ACM Reference Format:}

Jollen Chen, Dung-Cheng Lin, and Chuan-Hsiang Han. 2020. Volatility Effect on the Adoption and Valuation of Tokenomics. In The 35th ACM/SIGAPP Symposium on Applied Computing (SAC '20), March 30-April 3, 2020, Brno, Czech Republic. ACM, New York, NY, USA, Article 4, 7 pages. https://doi. org $/ 10.1145 / 3341105.3373891$

\section{INTRODUCTION}

Cryptographic (crypto for short) assets are based on blockchain technology such as consensus schemes on the distributed ledger. Cryptoassets might be described as either a coin or a token. The term coin generally expresses the payment purpose of an exchange medium, while token refers to an asset that gives the holder additional functionality or utility from a platform or ownership on the internet.

${ }^{*}$ Corresponding author. E-mail: chhan@mx.nthu.edu.tw Tel.:+ 886-3-5742224; fax: +886-3-5621823.

Permission to make digital or hard copies of all or part of this work for personal or classroom use is granted without fee provided that copies are not made or distributed for profit or commercial advantage and that copies bear this notice and the full citation on the first page. Copyrights for components of this work owned by others than ACM must be honored. Abstracting with credit is permitted. To copy otherwise, or republish, to post on servers or to redistribute to lists, requires prior specific permission and/or a fee. Request permissions from permissions@acm.org.

SAC '20, March 30-April 3, 2020, Brno, Czech Republic

(C) 2020 Association for Computing Machinery.

ACM ISBN 978-1-4503-6866-7/20/03 ..\$15.00

https://doi.org/10.1145/3341105.3373891
From the financial point of view, cryptoasset behaves a highly volatile pattern on its price dynamics, typically measured by volatility. A basic and simple notion of historical volatility means the (annualized) standard deviation of an asset's return data. There exists a huge literature in econometrics to study time-varying volatility models such as the discrete-time GRACH model [4] or the continuous-time instantaneous volatility model [10].

Based on empirical studies of historical asset prices presented in the Appendix of this paper, it is observed that medians of estimated volatilities from cryptoassets such as Bitcoin's BTCUSD and Ether's ETHUSD are $3 \sim 20$ times larger than those traditionally most-liquid ETFs (exchanged traded funds) such as GLD (commodity), SPY (equity index), FXE (foreign exchange), and AGG (bond). This finding indicates that the effect of volatility on cryptoassets dynamics can be significant despite that the impact of volatility on traditional assets has been recognized on the risk assessment and control, derivatives pricing, portfolio management, etc.

Several research works consider mechanism of the blockchain ecosystem and generalize classical economics models to incorporate the following effects: supply-demand interaction, e.g. Buchholz et al. [1], attractiveness for investors, e.g. Kristoufek [9], and global economic factors such as stock exchange indices, exchange rates, and oil prices, e.g. Van Wijk [12], etc.

It is documented that investor attractiveness is one of the most influential factors for the economy of cryptoassets. This type of research is currently active along the line of computer networks and social participation. Cong et al. [3] recently proposed a generic and dynamic model revealing a concrete relationship among the token value, the platform productivity, agent's optimal holding unit, adoption, user's transaction need, participation cost, and userbase. Their economic model is merely under the regime of constant volatility. Given the time-varying volatility characterized from observed crypto market prices, we extend models in [3] under time-varying volatility regime and focus its effect on token user adoption. We find that an intuition that enlarging the changes of platform productivity (usefulness) might increase the chance of token's high price turns out to be arguable, and we show that a highly volatile productivity behavior not only leads to the instability of userbase but also reduces the possibility for the platform to be fully adopted.

The userbase in tokenomics can be categorized into three cases: (i) counted by miners, (ii) counted by transactions, and (iii) counted by nodes of IoT devices. The functionality of IoT nodes is used merely for data transfer, which is essentially different from the first two cases. This paper further expands the original model [3] to 
meet the requirements of IoT blockchain network by adding the total amount of IoT nodes to userbase so that users, represented as IoT nodes, can join and execute transactions in this network.

The rest of this paper is organized as follows. Section 2 extends from the constant volatility regime in [3] to a general time-varying volatility model and presents the main results of tokenomics. Section 3 investigates a special case of IoT blockchain. Section 4 concludes and the financial data analytics is demonstrated in the appendix.

\section{TOKENOMICS UNDER DYNAMIC VOLATILITY MODEL}

In the section, motivated by the characteristics of token's price behavior shown in the Appendix, the objective is to generalize the original model proposed by Cong et al. [3] by releasing the constant volatility assumption and incorporating dynamic volatility.

First, the platform productivity process $A_{t}$ is modeled as follow:

$$
\frac{\mathrm{d} A_{t}}{A_{t}}=\mu^{A} \mathrm{~d} t+\sigma_{t}^{A} \mathrm{~d} Z_{t}^{A}
$$

Note that the original fixed volatility $\sigma^{A}$ used in [3] is changed into a stochastic volatility process $\sigma_{t}^{A}$.

Next, a conjecture about price $P_{t}$ is of the following form with a time-varying volatility $\sigma_{t}^{P}$ :

$$
\frac{\mathrm{d} P_{t}}{P_{t}}=\mu^{P} \mathrm{~d} t+\sigma_{t}^{P} \mathrm{~d} Z_{t}^{A}
$$

We remark that originally the drift of price in the paper is timedependent, and yet we find it unnecessary so that the drift here is only a positive constant.

Agents in the platform make a two-step decision (i) whether to incur a participation cost to join the platform, and if so, (ii) how many tokens to hold, which depends on both blockchain trade surplus and the expected future token price. Again, following similar argument in [3], the above two-step decision can be transformed into the following dynamic of cumulative payoff process $y_{i, t}$ (of agent $i$ in the index set $I=[0,1])$ :

$$
\begin{aligned}
\mathrm{d} y_{i, t}=\max \left\{0, \max _{k_{i, t}}\left[\left(P_{t} k_{i, t}\right)^{1-\alpha}\left(N_{t} A_{t} e^{u_{i}}\right)^{\alpha} \mathrm{d} t\right.\right. \\
\left.\left.\quad+k_{i, t} P_{t} \mu^{P} \mathrm{~d} t-\phi \mathrm{d} t-P_{t} k_{i, t} r \mathrm{~d} t\right]\right\}
\end{aligned}
$$

where $\left(P_{t} k_{i, t}\right)^{1-\alpha}\left(N_{t} A_{t} e^{u_{i}}\right)^{\alpha}$ is the utility flow with $k_{i, t}$ units of token held by agent $i$, userbase $N_{t}$, (heterogeneous) transaction need $e^{u_{i}}$ of agent $i ; \phi$ is the participation cost. This is so-called user-cost-of-capital argument [8].

If agent $i$ decides to join the platform, the optimal holding is given by F.O.C.:

$$
k_{i, t}^{*}=\frac{N_{t} A_{t} e^{u_{i}}}{P_{t}}\left(\frac{1-\alpha}{r-\mu^{P}}\right)^{\frac{1}{\alpha}},
$$

transforming the dynamic of $y_{i, t}$ into

$$
\mathrm{d} y_{i, t}=\max \left\{0, N_{t} A_{t} e^{u_{i}} \alpha\left(\frac{1-\alpha}{r-\mu^{P}}\right)^{\frac{1-\alpha}{\alpha}}-\phi\right\} \mathrm{d} t .
$$

On the one hand, the (minimal) threshold $\underline{u}_{t}$ of agent's transaction need to participate in the platform is then given by

$$
\underline{u}_{t}\left(N_{t} ; A_{t}, \mu^{P}\right)=-\ln \left(N_{t}\right)+\ln \left(\frac{\phi}{A_{t} \alpha}\right)-\frac{1-\alpha}{\alpha} \ln \left(\frac{1-\alpha}{r-\mu^{P}}\right) .
$$

On the other hand, since only users with $u_{i} \geq \underline{u}_{t}$ will join the platform, one has

$$
N_{t}=1-G\left(\underline{u}_{t}\right)
$$

where $G(\cdot)$ is the cumulative distribution function (CDF) of random variable $u_{i}$. Combining (4) and (5), one can try to simultaneously solve equilibriums $\underline{u}_{t}$ and $N_{t}$. More precisely, substituting $N_{t}$ in (4) by (5) we have

$$
\underline{u}_{t}=-\ln \left(1-G\left(\underline{u}_{t}\right)\right)+\ln \left(\frac{\phi}{A_{t} \alpha}\right)-\frac{1-\alpha}{\alpha} \ln \left(\frac{1-\alpha}{r-\mu^{P}}\right) \triangleq h\left(\underline{u}_{t}\right) .
$$

Its solution $\underline{u}_{t}^{*}$ can be solved by the fixed point of the following iterations ${ }^{1}$ :

$$
\underline{u}_{t, i+i}=h\left(\underline{u}_{t, i}\right), \quad \underline{u}_{t, 0}=u_{0}
$$

Note that conditioning on the productivity $A_{t}$ and given the growth rate $\mu^{P}$ of token price, we can always solve a corresponding non-trivial solution $\left(\underline{u}_{t}^{*}, N_{t}^{*}\right)$, by observing that as the platform productivity $A_{t}$ increases, the corresponding userbase $N_{t}^{*}$ increases and yet the threshold of the transaction need $\underline{u}_{t}^{*}$ decreases. This qualitative property leads to an extremal case when the productivity is extremely expanded $A_{t} \rightarrow \infty$, so that the corresponding threshold diminishes $\underline{u}_{t}^{*} \rightarrow-\infty$ (see (4)) and the userbase is fully adopted $N_{t}^{*} \rightarrow 1$ (see (5)). In the following section, we will abuse the use of equilibrium $\left(\underline{u}_{t}^{*}, N_{t}^{*}\right)$ by merely $\left(\underline{u}_{t}, N_{t}\right)$ for simplicity.

\subsection{Userbase Adoption: Volatile Productivity Causes Volatile Userbase}

Given the aforementioned observation, one may suspect that higher volatility of productivity to be a bless (since high volatility makes it easier to obtain large $A_{t}$, low threshold $\underline{u}_{t}$ (cf. (4)), and thus large $N_{t}$ (cf. (5)). In fact, we will show later that higher volatility (of productivity) leads to larger userbase $N_{t}$ is merely a misunderstanding through a detailed analysis below.

We explain this misunderstanding by raising the following two questions:

(Q1) How does productivity volatility affects userbase volatility?

(Q2) How does productivity volatility affects user adoption?

The answer of (Q1) is provided in the following proposition.

Proposition 2.1 (Threshold Dynamic). The volatility of threshold $\underline{u}_{t}$ is controlled by its martingale term

$$
\left(\frac{g\left(\underline{u}_{t}\right)\left(1-G\left(\underline{u}_{t}\right)\right)}{1-G\left(\underline{u}_{t}\right)-g\left(\underline{u}_{t}\right)}\right) \sigma_{t}^{A} \mathrm{~d} Z_{t}^{A}
$$

Hence, high volatility $\sigma_{t}^{A}$ of platform productivity amplifies the volatility of $\underline{u}_{t}$.

${ }^{1}$ The existence of the solution is proved in [3]. 
Proof. The calculation of the dynamic of $\underline{\mathrm{d}}_{t}$ is as follows.

$$
\begin{aligned}
\mathrm{d} \underline{u}_{t}= & \mathrm{d}\left(-\ln \left(1-G\left(\underline{u}_{t}\right)\right)+\ln \left(\frac{\phi}{A_{t} \alpha}\right)-\frac{1-\alpha}{\alpha} \ln \left(\frac{1-\alpha}{r-\mu^{P}}\right)\right) \\
= & \mathrm{d}\left(-\ln \left(1-G\left(\underline{u}_{t}\right)\right)-\ln A_{t}\right) \\
= & \frac{g\left(\underline{u}_{t}\right)}{1-G\left(\underline{u}_{t}\right)} \mathrm{d} \underline{u}_{t}+\frac{1}{2}\left(\frac{g^{\prime}\left(\underline{u}_{t}\right)}{1-G\left(\underline{u}_{t}\right)}+\frac{g^{2}\left(\underline{u}_{t}\right)}{\left(1-G\left(\underline{u}_{t}\right)\right)^{2}}\right) \mathrm{d}\left\langle\underline{u}_{t}\right. \\
& \quad-\frac{1}{A_{t}} \mathrm{~d} A_{t}+\frac{1}{2} \frac{1}{A_{t}^{2}} \mathrm{~d}\langle A\rangle_{t}
\end{aligned}
$$

Rewrite the above equation, we have

$$
\begin{aligned}
& \left(1-\frac{g\left(\underline{u}_{t}\right)}{1-G\left(\underline{u}_{t}\right)}\right) \mathrm{d} \underline{u}_{t} \\
& =\left[\frac { 1 } { 2 } ( \frac { g ^ { \prime } ( \underline { u } _ { t } ) } { 1 - G ( \underline { u } _ { t } ) } + \frac { g ^ { 2 } ( \underline { u } _ { t } ) } { ( 1 - G ( \underline { u } _ { t } ) ) ^ { 2 } } ) \mathrm { d } \left\langle\underline{u}_{t}\right.\right. \\
& \left.\quad+\left(\frac{1}{2}\left(\sigma_{t}^{A}\right)^{2}-\mu^{A}\right) \mathrm{d} t\right]+\sigma_{t}^{A} \mathrm{~d} Z_{t}^{A} .
\end{aligned}
$$

Note that the right hand side of (6) involves the quadratic variation $\langle\underline{u}\rangle_{t}$ of $\underline{u}_{t}$, which can be solved by calculating again the quadratic variation of this equation.

$$
\left(1-\frac{g\left(\underline{u}_{t}\right)}{1-G\left(\underline{u}_{t}\right)}\right)^{2} \mathrm{~d}\left\langle\underline{u}_{t}=\left(\sigma_{t}^{A}\right)^{2} \mathrm{~d} t,\right.
$$

or, equivalently,

$$
\mathrm{d}\left\langle\underline{u}_{t}=\left(1-\frac{g\left(\underline{u}_{t}\right)}{1-G\left(\underline{u}_{t}\right)}\right)^{-2}\left(\sigma_{t}^{A}\right)^{2} \mathrm{~d} t .\right.
$$

Plugging (7) into (6) yields

$$
\begin{aligned}
\mathrm{d} \underline{u}_{t}=\left(\frac { 1 } { 2 } ( 1 - \frac { g ( \underline { u } _ { t } ) } { 1 - G ( \underline { u } _ { t } ) } ) ^ { - 3 } \left(1+\frac{g^{\prime}\left(\underline{u}_{t}\right)}{1-G\left(\underline{u}_{t}\right)}\right.\right. & \\
& \left.\left.+\frac{g^{2}\left(\underline{u}_{t}\right)}{\left(1-G\left(\underline{u}_{t}\right)\right)^{2}}\right)\left(\sigma_{t}^{A}\right)^{2}-\mu^{A}\right) \mathrm{d} t+\sigma_{t}^{A} \mathrm{~d} Z_{t}^{A} .
\end{aligned}
$$

Moreover, from (5) we get $\mathrm{d} N_{t}=-g\left(\underline{u}_{t}\right) \mathrm{d} \underline{u}_{t}-\frac{1}{2} g^{\prime}\left(\underline{u}_{t}\right) \mathrm{d}\langle\underline{u}\rangle_{t}$, and thus the drift term and martingale term of userbase dynamic are given respectively by

$$
\begin{array}{r}
\left\{-\frac{1}{2}\left(\sigma_{t}^{A}\right)^{2}\left[g ( \underline { u } _ { t } ) ( 1 - \frac { g ( \underline { u } _ { t } ) } { 1 - G ( \underline { u } _ { t } ) } ) ^ { - 3 } \left(1+\frac{g^{\prime}\left(\underline{u}_{t}\right)}{1-G\left(\underline{u}_{t}\right)}\right.\right.\right. \\
\left.\left.+\frac{g^{2}}{\left(1-G\left(\underline{u}_{t}\right)\right)^{2}}\right)+g^{\prime}\left(\underline{u}_{t}\right)\left(1-\frac{g\left(\underline{u}_{t}\right)}{1-G\left(\underline{u}_{t}\right)}\right)^{-2}\right] \\
\left.+g\left(\underline{u}_{t}\right) \mu^{A}\right\} \mathrm{d} t
\end{array}
$$

and

$$
\left(\frac{g\left(\underline{u}_{t}\right)\left(1-G\left(\underline{u}_{t}\right)\right)}{1-G\left(\underline{u}_{t}\right)-g\left(\underline{u}_{t}\right)}\right) \sigma_{t}^{A} \mathrm{~d} Z_{t}^{A} .
$$

To conclude, the above martingale term (10) suggests that even if the productivity has more chances to grow high when $\sigma_{t}^{A}$ grows, the corresponding userbase volatility also increases. Meanwhile, equation (9) indicates that the drift term usually decreases as $\sigma_{t}^{A}$ increases and/or $\mu^{A}$ decreases because generally low threshold ${ }^{2}$, i.e. $\underline{u}_{t} \ll 0$, has $g\left(\underline{u}_{t}\right) \rightarrow 0$ with $g^{\prime}\left(\underline{u}_{t}\right)>0$ and $G\left(\underline{u}_{t}\right) \rightarrow 0$. In fact, we only need $0<g\left(\underline{u}_{t}\right)<1,1-G\left(\underline{u}_{t}\right)>0$ and $g^{\prime}\left(\underline{u}_{t}\right)>0$. by taking the standard normal random variable same as in [3], the above conditions is fulfilled when $\underline{u}_{t}<0$.

Next, the attempt to answer (Q2) is more sophisticated and the conclusion is put in the following theorem.

THEOREM 2.2 (BoundS For USERBASE UPPER TAIL PROBABILITY). Under the fast mean-reverting assumption of $\sigma_{t}^{A}$, the possibility for platform achieving high adoption is dampened by high productivity volatility. Mathematically, the claim can be formulated as follows.

$$
\begin{aligned}
\Phi\left(\frac{G^{-1}(\varepsilon)+\mu^{A} t}{\overline{\sigma^{A}} \sqrt{t}}-M \overline{\sigma^{A}} \sqrt{t}\right) \leq & \mathbb{P}\left(N_{t} \geq 1-\varepsilon\right) \\
& \leq \Phi\left(\frac{G^{-1}(\varepsilon)+\mu^{A} t}{\overline{\sigma^{A}} \sqrt{t}}-m \overline{\sigma^{A}} \sqrt{t}\right),
\end{aligned}
$$

where $\overline{\sigma^{A}}$ is the effective volatility and $m$ and $M$ are some positive constants.

Proof. For an arbitrary small $0<\varepsilon \ll 1$, we exploit the volatility effect on user adoption by estimating the upper tail probability for $N_{t}$ :

$$
\mathbb{P}\left(N_{t} \geq 1-\varepsilon\right),
$$

From (5), it can be transformed as

$$
\mathbb{P}\left(1-G\left(\underline{u}_{t}\right) \geq 1-\varepsilon\right) \text {, or } \mathbb{P}\left(G\left(\underline{u}_{t}\right) \leq \varepsilon\right) .
$$

For $G(\cdot)$ being a CDF (and thus monotonic), its inverse function $G^{-1}$ is assumed uniquely exist so that (11) becomes a lower tail estimation for $\underline{u}_{t}$ :

$$
\mathbb{P}\left(\underline{u}_{t} \leq G^{-1}(\varepsilon)\right) .
$$

Now, we can apply some asymptotic analysis [5] to estimate the above probability.

First, for $\varepsilon>0$ small enough, there exists a constant $m>0$ (resp. $M>0$ ) such that the process (8) is bounded below (resp. bounded above) by

$$
\begin{gathered}
\mathrm{d} v_{t}=\left(m\left(\sigma_{t}^{A}\right)^{2}-\mu^{A}\right) \mathrm{d} t+\sigma_{t}^{A} \mathrm{~d} Z_{t}^{A} . \\
\text { (resp. } \left.\mathrm{d} v_{t}=\left(M\left(\sigma_{t}^{A}\right)^{2}-\mu^{A}\right) \mathrm{d} t+\sigma_{t}^{A} \mathrm{~d} Z_{t}^{A}\right)
\end{gathered}
$$

Thus, we are able to find an upper bound (resp. lower bound) for (12):

$$
\begin{gathered}
\mathbb{P}\left(\underline{u}_{t} \leq G^{-1}(\varepsilon)\right) \leq \mathbb{P}\left(v_{t} \leq G^{-1}(\varepsilon)\right) \\
\text { (resp. } \left.\mathbb{P}\left(\underline{u}_{t} \leq G^{-1}(\varepsilon)\right) \geq \mathbb{P}\left(v_{t} \leq G^{-1}(\varepsilon)\right)\right)
\end{gathered}
$$

Next, under the assumption that the stochastic volatility $\sigma_{t}^{A}$ is governed by a fast mean-reverting process, the homogenized dynamics of (13) takes the asymptotics $\overline{\sigma^{A}}$ as the effective volatility of $v_{t}$, see [5] for further details. Then

$$
\begin{aligned}
& \overline{v_{t}}=\left(m\left(\overline{\sigma^{A}}\right)^{2}-\mu^{A}\right) t+\overline{\sigma^{A}} Z_{t}^{A} \sim \mathcal{N}\left(\left(m\left(\overline{\sigma^{A}}\right)^{2}-\mu^{A}\right) t,\left(\overline{\sigma^{A}}\right)^{2} t\right) . \\
& \left(\text { resp. } \overline{v_{t}}=\left(M\left(\overline{\sigma^{A}}\right)^{2}-\mu^{A}\right) t+\overline{\sigma^{A}} Z_{t}^{A} \sim \mathcal{N}\left(\left(M\left(\overline{\sigma^{A}}\right)^{2}-\mu^{A}\right) t,\left(\overline{\sigma^{A}}\right)^{2} t\right)\right)
\end{aligned}
$$

${ }^{2}$ We put emphasis on the situation of low threshold that allows more people to join the platform, (12) is of the same concern. 
Finally, one can see that

$$
\begin{aligned}
& \mathbb{P}\left(v_{t} \leq G^{-1}(\varepsilon)\right)=\mathbb{P}\left(\overline{v_{t}} \leq G^{-1}(\varepsilon)\right)+O(\delta) \\
= & \mathbb{P}\left(\frac{\overline{v_{t}}-\left(m\left(\overline{\sigma^{A}}\right)^{2}-\mu^{A}\right) t}{\overline{\sigma^{A}} \sqrt{t}} \leq \frac{G^{-1}(\varepsilon)-\left(m\left(\overline{\sigma^{A}}\right)^{2}-\mu^{A}\right) t}{\overline{\sigma^{A}} \sqrt{t}}\right)+O(\delta) \\
= & \Phi\left(\frac{G^{-1}(\varepsilon)+\mu^{A} t}{\overline{\sigma^{A}} \sqrt{t}}-m \overline{\sigma^{A}} \sqrt{t}\right)+O(\delta),
\end{aligned}
$$

where the parameter $\delta \ll 1$ refers to a small time scale of the driving volatility process, and $\Phi$ remains as $\operatorname{CDF}$ of $\mathcal{N}(0,1)$. This leads to

$$
\begin{gathered}
\mathbb{P}\left(N_{t} \geq 1-\varepsilon\right) \leq \Phi\left(\frac{G^{-1}(\varepsilon)+\mu^{A} t}{\overline{\sigma^{A}} \sqrt{t}}-m \overline{\sigma^{A}} \sqrt{t}\right)+O(\delta) . \\
\text { (resp. } \left.\mathbb{P}\left(N_{t} \geq 1-\varepsilon\right) \geq \Phi\left(\frac{G^{-1}(\varepsilon)+\mu^{A} t}{\overline{\sigma^{A}} \sqrt{t}}-M \overline{\sigma^{A}} \sqrt{t}\right)+O(\delta)\right)
\end{gathered}
$$

Above equations indicate the inverse effect between $\sigma_{t}^{A}$ and $\mathbb{P}\left(N_{t} \geq 1-\varepsilon\right)$. Namely, higher volatility of productivity results in lower chance for high adoption, or equivalently, user adoption of the platform benefits from its steadily increasing productivity.

In sum, it follows from (5) that to decrease the threshold $\underline{u}_{t}$ (and thus increase the token price as seen later in (14)), one can either let $\mu^{P}$ approach the risk-free rate $r$ or increase the value of productivity $A_{t}$, the only exogenous factor. In practice, the former is uncontrollable, leading us to study the effect of the latter. We prove that highly volatile behavior of $A_{t}$ is in general unfavorable for not only creating undesirable fluctuations for userbase but also diminishing the chance of userbase under high adoption. Instead, the steadily increasing growth rate of platform's usefulness is beneficial in the long run.

\subsection{Token Valuation}

Assume the total token supply is fixed at $M=\int_{0}^{1} k_{i, t} \mathrm{~d} i$ and denote the aggregated participants' transaction need as

$$
S_{t}=\int_{\underline{u}_{t}}^{\infty} e^{u} g(u) \mathrm{d} u
$$

where $g(\cdot)$ is the probability density function (pdf) of random variable $u_{i}$. By integrating over $I$, (3) becomes

$$
P_{t}=\frac{N_{t} S_{t} A_{t}}{M}\left(\frac{1-\alpha}{r-\mu^{P}}\right)^{\frac{1}{\alpha}} .
$$

The Markov equilibrium with $A_{t}$ (i.e. $P_{t}=P\left(A_{t}\right)$ ) can be solved as follows.

By Ito lemma

$$
\mathrm{d} P_{t}=\mathrm{d} P\left(A_{t}\right)=P^{\prime}\left(A_{t}\right) \mathrm{d} A_{t}+\frac{1}{2} P^{\prime \prime}\left(A_{t}\right) \mathrm{d}\langle A\rangle_{t} .
$$

Comparing the coefficient of $\mathrm{d} P_{t}$ with the conjecture in (1), we have

$$
P^{\prime}\left(A_{t}\right) \mu^{A} A_{t}+\frac{1}{2} P^{\prime \prime}\left(A_{t}\right)\left(\sigma_{t}^{A}\right)^{2} A_{t}^{2}=\mu^{P} P_{t}
$$

and

$$
P^{\prime}\left(A_{t}\right) \sigma_{t}^{A} A_{t}=\sigma_{t}^{P} P_{t}
$$

An interesting observation is the case of full adoption (i.e. $N_{t}=1$ ). Let's first denote

$$
\bar{S}=\int_{-\infty}^{\infty} e^{u} g(u) \mathrm{d} u,
$$

then the pricing formula (14) under full adoption becomes

$$
\bar{P}_{t}=\frac{A_{t} \bar{S}}{M}\left(\frac{1-\alpha}{r-\mu^{P}}\right)^{\frac{1}{\alpha}} .
$$

This immediately leads to $P^{\prime}\left(A_{t}\right)=\frac{\bar{S}}{M}\left(\frac{1-\alpha}{r-\mu^{P}}\right)^{\frac{1}{\alpha}}$ and thus (16) gives $\sigma_{t}^{A}=\sigma_{t}^{P}$. Similarly, (15) implies $\mu^{A}=\mu^{P}$. This is consistent with (17) that $P_{t} \propto A_{t}$.

In sum, the dynamic of token price is shown to be

$$
P_{t}=\frac{N_{t} S_{t} A_{t}}{M}\left(\frac{1-\alpha}{r-\mu^{P}}\right)^{\frac{1}{\alpha}}
$$

and is determined by userbase $N_{t}$, aggregated transactional need $S_{t}$, platform productivity $A_{t}$, total token supply $M$ and expected token appreciation $\mu^{P}$. The limiting behavior of price $P_{t}$ (under full adpotion) is reduced to (17).

\section{CASE STUDY: TOKENOMICS OF IoT BLOCKCHAIN}

In this section, we would like to generalize previous results for IoT Blockchain. Unlike miners and transactions, the usage of IoT devices is for transferring data and these devices interconnect heterogeneously to a system. For each IoT node, it participates a system by bootstrap then enters into a peer-to-peer network by stabilization algorithms to overcome the collective effect, i.e., churn from the group level. For example, Flowchain [2] applies the finger table technique for latency measurement so as to periodically determine whether a node stays in the peer level or not.

We postulate that the platform of IoT blockchain does not incur any participation fee, i.e. $\phi=0$. Thus the previous two-step decision (2) can be simplified by

$$
\begin{aligned}
\mathrm{d} y_{i, t}=\max _{k_{i, t}}\left(\left(P_{t} k_{i, t}\right)^{1-\alpha}\left(N_{t} A_{t} e^{u_{i}}\right)^{\alpha}\right. & \\
& \left.+k_{i, t} P_{t} \mu^{P}-P_{t} k_{i, t} r\right) \mathrm{d} t .
\end{aligned}
$$

Following the same argument of (3), the optimal holding is given by

$$
k_{i, t}^{*}=\frac{N_{t} A_{t} e^{u_{i}}}{P_{t}}\left(\frac{1-\alpha}{r-\mu^{P}}\right)^{\frac{1}{\alpha}} .
$$

Secondly, the setting for userbase $N_{t}$ should be modified because the fluctuation of $N_{t}$ for IoT blockchain is much more severe than that of traditional ones. For this particular feature of IoT blockchain, we model the dynamic of userbase $N_{t}$ as a Jacobi process ${ }^{3}$

$$
\mathrm{d} N_{t}=\kappa\left(m-N_{t}\right) \mathrm{d} t+\xi \sqrt{N_{t}\left(1-N_{t}\right)} \mathrm{d} Z_{t}^{A},
$$

where model parameters $(\kappa, m, \xi)$ denote the mean-reverting rate, long-run mean, and volatility of volatility.

${ }^{3}$ The stochastic process $N_{t}$ is bounded in interval $(0,1)$ provided $\kappa \geq \frac{\xi}{1 \pm(2 m-1)}$. 
Hence, under the assumption of fixed total token supply $M$, the aggregated participants' transaction need is given by

$$
\bar{S}=\int_{-\infty}^{\infty} e^{u} g(u) \mathrm{d} u
$$

as there is no minimal threshold. Combing these results, we again obtain the pricing formula (cf. (14))

$$
P_{t}=\frac{N_{t} \bar{S} A_{t}}{M}\left(\frac{1-\alpha}{r-\mu^{P}}\right) \triangleq C N_{t} A_{t} .
$$

By Ito lemma, the dynamic of the token price is as follows.

$$
\begin{aligned}
\mathrm{d} P_{t}= & C \mathrm{~d}\left(N_{t} A_{t}\right) \\
= & C\left(N_{t} \mathrm{~d} A_{t}+A_{t} \mathrm{~d} N_{t}+\mathrm{d} A_{t} \mathrm{~d} N_{t}\right) \\
= & C A_{t}\left(N_{t} \mu^{A}+\kappa\left(m-N_{t}\right)+\sigma_{t}^{A} \xi \sqrt{N_{t}\left(1-N_{t}\right)}\right) \mathrm{d} t \\
& \quad+C A_{t}\left(N_{t} \sigma_{t}^{A}+\xi \sqrt{N_{t}\left(1-N_{t}\right)}\right) \mathrm{d} Z_{t}^{A} .
\end{aligned}
$$

It follows from (19) that

$$
\begin{aligned}
\frac{\mathrm{d} P_{t}}{P_{t}}=\left(\mu^{A}+\kappa \frac{m-N_{t}}{N_{t}}+\sigma_{t}^{A}\right. & \left.\frac{\sqrt{N_{t}\left(1-N_{t}\right)}}{N_{t}}\right) \mathrm{d} t \\
& +\left(\sigma_{t}^{A}+\xi \frac{\sqrt{N_{t}\left(1-N_{t}\right)}}{N_{t}}\right) \mathrm{d} Z_{t}^{A}
\end{aligned}
$$

This modification allows the userbase to be more fluctuating than the usual one, and meanwhile preserves most of the previous theoretical properties. Obviously the pricing formula (19) remains the same (except for $S_{t}$ replaced by $\bar{S}$ ). Accordingly, the limiting behavior of token price (under full adoption) also remains the same, i.e., same drift and volatility. This can be seen from (19) that as $N_{t} \rightarrow 1, P_{t}$ is proportional to $A_{t}$.

The intuition that from (19) that as $N_{t} \rightarrow 1, P_{t}$ is proportional to $A_{t}$ is arguable. The correction deduction from (20) discloses as $N_{t} \rightarrow 1$, the productivity dynamics becomes $\frac{\mathrm{d} P_{t}}{P_{t}}=$ $\left(\mu^{A}+\kappa(m-1)\right) \mathrm{d} t+\sigma_{t}^{A} \mathrm{~d} Z_{t}^{A}$; the drift term is different from that of $A_{t}$. Such "inconsistency" arises from our selection of stochastic model for userbase $N_{t}$. Note that the Jacobi process in (18) is a mean-reverting process, which naturally implies that the equilibrium of $N_{t}$ is the long run mean $m$. In other words, as we take $N_{t} \rightarrow 1$, there is a natural resistance from the driving force $Z_{t}^{A}$. Thus, the deduction from (20) is in fact consistent with that from (19).

Also, one can readily observe that in general volatility term of token price $P_{t}$ is larger than that of productivity $A_{t}$ (i.e. $\sigma_{t}^{A}+$ $\left.\xi \sqrt{N_{t}\left(1-N_{t}\right)} / N_{t}>\sigma_{t}^{A}\right)$.

Note that since the IoT blockchain is a decentralized peer-to-peer network, the need to further establish an incentive scheme might be crucial to build a sustainable IoT network. We will leave this aspect as a research topic in the future.

\section{CONCLUSIONS}

Based on the characteristics from observed token asset prices, we generalize Cong et. al. [3] by incorporating time-varying volatility and investigate its effect on the adoption and valuation of tokenomics. We find that high userbase adoption and stable token price are associated with volatility reduction. Since the IoT blockchain can be viewed as a special case of our generalized model, we also find that the token price is often more volatile than productivity process, and token price can be stabilized by the productivity volatility and the userbase adoption. In the future, we plain to tackle challenges of missing IoT data and empirical estimation for the generalized model.

\section{A COMPARISONS OF DYNAMIC VOLATILITY ESTIMATES FOR TRADITIONAL ASSETS AND CRYPTOASSETS}

\section{A.1 Volatility Estimations}

The estimation of volatility $\sigma_{t}^{P}$ can be categorized into the discretetime GARCH model, and the continuous-time instantaneous volatility model as follows.

1. GARCH $(1,1)$ model [4]:

The $\mathcal{F}_{t-1}$-conditional return $r_{t}$ is modeled by its growth rate $\mu_{t}$

$$
r_{t} \mid \mathcal{F}_{t-1}=\mu_{t}+\sigma_{t}^{P} \varepsilon_{t}, \quad \varepsilon_{t} \sim N(0,1)
$$

and the conditional volatility $\sigma_{t}^{P}$ (or, for simplicity, $\sigma_{t}$ ) is calculated recursively by

$$
\sigma_{t}^{2}=\gamma \bar{\sigma}+\alpha r_{t-1}^{2}+\beta \sigma_{t-1}^{2}, \quad \alpha+\beta+\gamma=1,
$$

where $\bar{\sigma}$ is the long run mean, parameters $(\alpha, \beta, \gamma)$ are proper coefficients. The estimation for these parameters can be seen in [11] or [7].

2. Fourier transform [10]:

Assume the log price $u_{t}$ satisfies a semi-martingale defined by $\mathrm{d} u_{t}=\mu_{t} \mathrm{~d} t+\sigma_{t} \mathrm{~d} W_{t}$. Then the instantaneous volatility is shown to satisfy

$$
\sigma^{2}(t)=2 \pi \mathfrak{F}^{-1}\left(\left(\mathfrak{F}(\mathrm{d} u) *_{B} \mathfrak{F}(\mathrm{d} u)\right)(k)\right)
$$

where $\mathfrak{F}(\mathrm{d} u)(k):=\frac{1}{2 \pi} \int_{0}^{2 \pi} e^{-i k t} \mathrm{~d} u_{t}$ and $*_{B}$ denotes the Bohr convolution product:

$$
\Phi *_{B} \Psi:=\lim _{N \rightarrow \infty} \frac{1}{2 N+1} \sum_{s=-N}^{N} \Phi(s) \Psi(k-s) .
$$

The estimation for parameters can be seen in [6].

\section{A.2 Empirical Results}

From the following Figure 1-Figure 6, higher outlier volatility values can be seen on Bitcoin (BTC) and Ether (ETH) than on traditional assets, for which four ETFs - GLD (commodity), SPY (equity), FXE (foreign exchange), and AGG (bond) - are taken as bench examples. In each figure, estimated volatilities are listed on left y-axis and the asset price is listed on the right y-axis.

We remark that, in what follows, navy line is the index; red line is conditional volatility $\sigma_{t}^{P}$ calculated by $\operatorname{GARCH}(1,1)$ as mentioned above; yellow line is the historical volatility (calculated from the standardized log return of assets); green line is the instantaneous volatility (or instant volatility for short) by Fourier transform. 


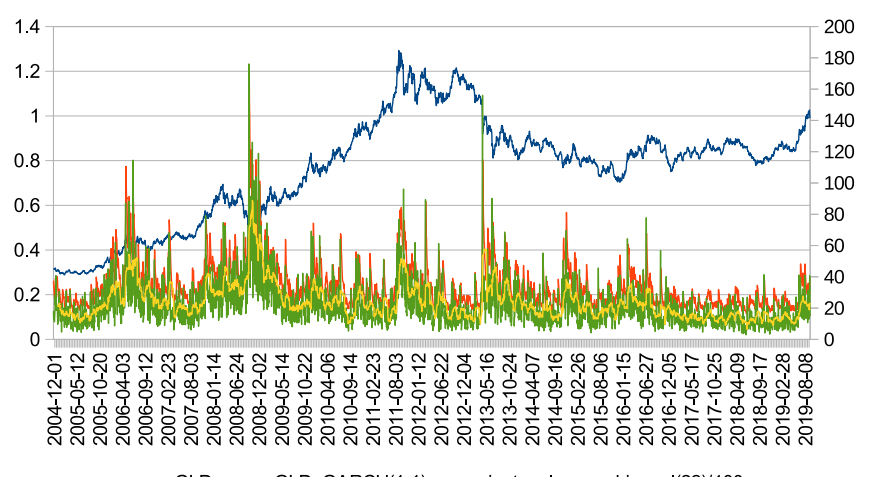

Figure 1: Volatility estimations for GLD (commodity) ETF.

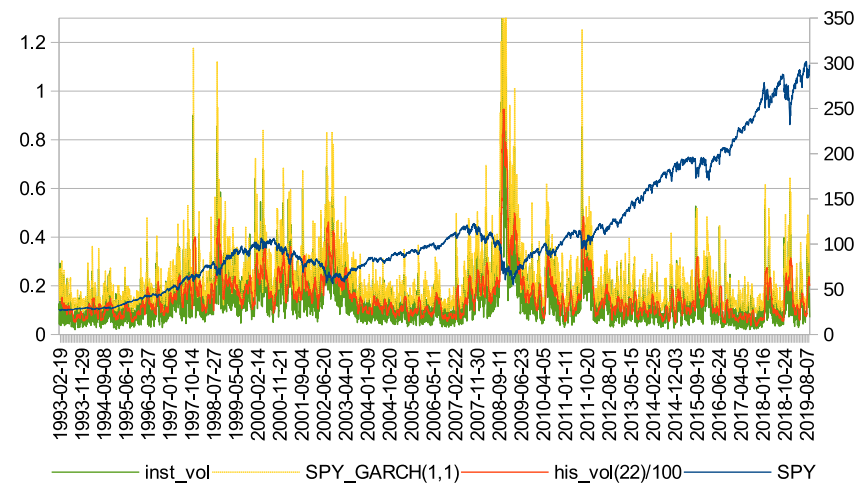

Figure 2: Volatility estimations for SPY (equity index) ETF.

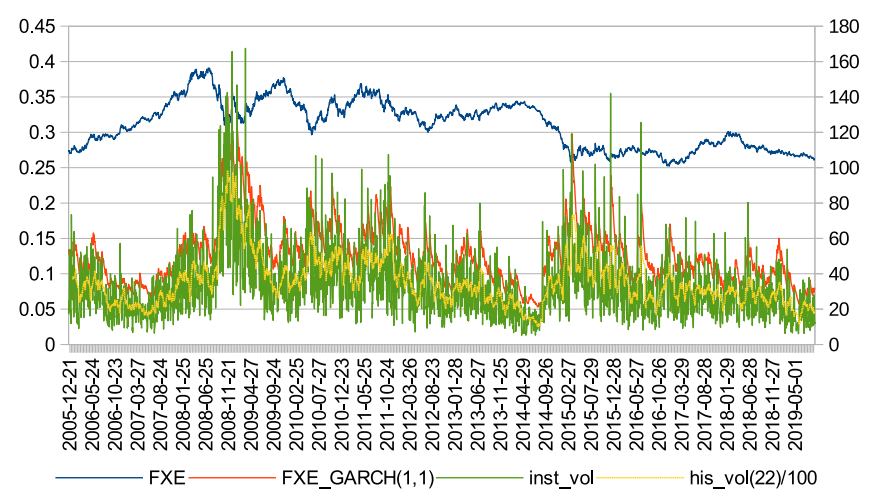

Figure 3: Volatility estimations for FXE (foreign exchange) ETF.

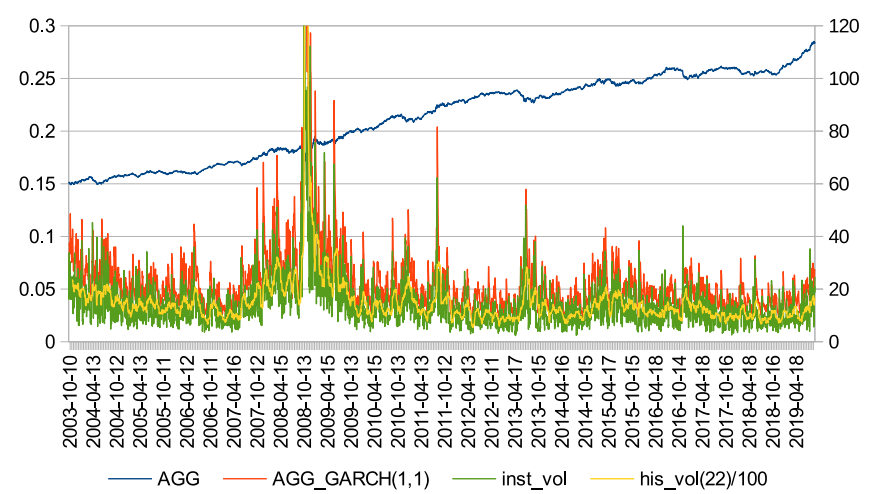

Figure 4: Volatility estimations for AGG (bond) ETF.

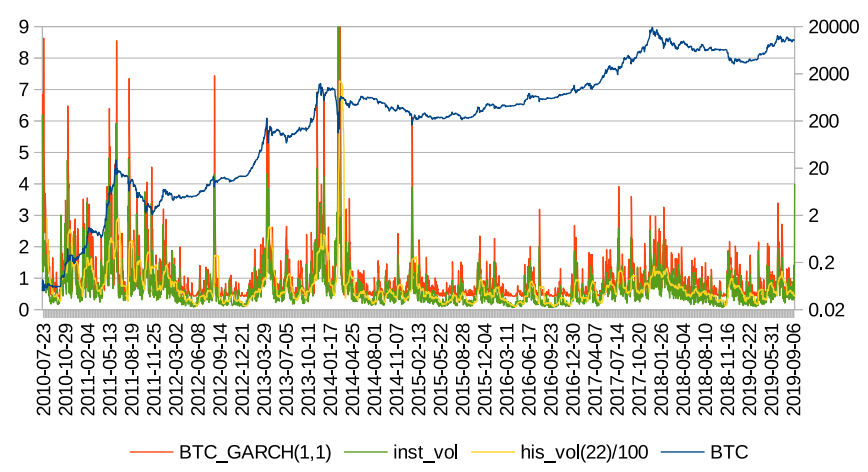

Figure 5: Volatility estimations for Bitcoin (BTC/USD).

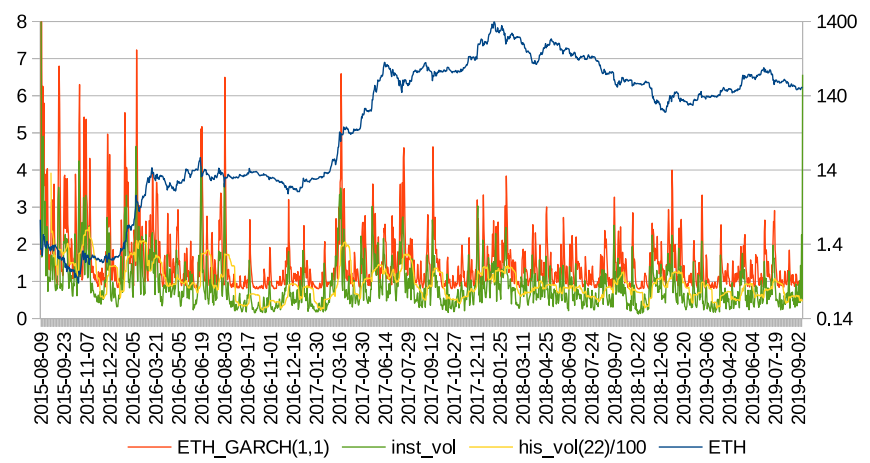

Figure 6: Volatility estimations for Ether (ETH/USD). 
Furthermore, to understand the volatilities apart from the outliers, their medians are also compared in Table 1. The increasing order of volatility medians is AGG (bond), FXE (foreign exchange), SPY (equity), GLD (commodity), BTC, and ETH. Cryptoassets are more volatile than traditional assets.

\begin{tabular}{|c|c|c|c|}
\hline & GARCH(1,1) & 22-day historical volatility & Instant volatility \\
\hline \hline GLD & 0.2139 & 0.1473 & 0.1306 \\
\hline SPY & 0.1909 & 0.1323 & 0.1170 \\
\hline FXE & 0.1223 & 0.0822 & 0.0734 \\
\hline AGG & 0.0491 & 0.0327 & 0.0296 \\
\hline BTC & $\mathbf{0 . 7 5 4 9}$ & $\mathbf{0 . 5 7 4 7}$ & $\mathbf{0 . 4 7 5 7}$ \\
\hline ETH & $\mathbf{1 . 2 1 4 1}$ & $\mathbf{0 . 8 8 4 1}$ & $\mathbf{0 . 7 0 8 6}$ \\
\hline
\end{tabular}

Table 1: The median volatilities of the ETFs and cryptoassets.

\section{B GENERAL NOTATIONS}

\begin{tabular}{ll} 
Notation & Definition \\
\hline \hline$A_{t}$ & platform productivity \\
$\mu^{A}$ & drift term of dynamic of $A$ \\
$\sigma_{t}^{A}$ & martingale term of dynamic $A$ \\
$Z_{t}^{A}$ & driving Brownian motion of dynamic $A$ \\
$M$ & total token supply \\
$P_{t}$ & token price \\
$\bar{P}_{t}$ & token price under full adoption \\
$e^{u_{i}}$ & transaction need of agent $i$ \\
$S_{t}$ & aggregated users' transaction need \\
$\bar{S}$ & aggregated users' transaction need under full adoption \\
$N_{t}$ & userbase \\
$y_{i, t}$ & cumulative payoff \\
$k_{i, t}$ & agent $i$ 's token holding \\
$k_{i, t}^{*}$ & agent $i$ 's optimal token holding \\
$\phi$ & participation cost \\
$\alpha$ & a constant between $(0,1)$ \\
$r$ & risk-free interest rate \\
\hline$\sigma^{A}$ & effective volatility of $\sigma_{t}^{A}$ \\
$\underline{u}_{t}$ & minimum threshold to join the platform \\
$g(u)$ & pdf of random variable $U$ at $U=u$ \\
$G(u)$ & CDF of random variable $U$ at $U=u$ \\
$\langle X\rangle$ & quadratic variation of $X$ \\
$\mathcal{N}\left(\mu, \sigma^{2}\right)$ & normal distribution with mean $\mu$ and variance $\sigma^{2}$ \\
$\Phi$ & CDF of $\mathcal{N}(0,1)$ \\
$\kappa$ & mean reverting rate of Jacobi process \\
$m$ & long run mean of Jacobi process \\
$\xi$ & volatility of volatility of Jacobi process \\
$\mathcal{F}(f)$ & Fourier transform of $f$ \\
$\mathcal{F}^{-1}(f)$ & inverse Fourier transform of $f$ \\
$* B$ & Bohr convolution product \\
&
\end{tabular}

\section{REFERENCES}

[1] Martis Buchholz, Jess Delaney, Joseph Warren, and Jeff Parker. 2012. Bits and bets, information, price volatility, and demand for Bitcoin. Economics 312 (2012), $2-48$.
[2] Jollen Chen. 2019. Flowchain white paper. (2019). https://flowchain.co/ Flowchain-Whitepaper-3.0-en.pdf

[3] Lin William Cong, Ye Li, and Neng Wang. 2019. Tokenomics: Dynamic adoption and valuation. Becker Friedman Institute for Research in Economics Working Paper (2019).

[4] Robert F. Engle and Kenneth F. Kroner. 1995. Multivariate simultaneous generalized ARCH. Econometric theory 11, 1 (1995), 122-150.

[5] Jean-Pierre Fouque, George Papanicolaou, Ronnie Sircar, and Knut Sølna. 2011. Multiscale stochastic volatility for equity, interest rate, and credit derivatives. Cambridge University Press.

[6] Chuan-Hsiang Han, Wei-Han Liu, and Tzu-Ying Chen. 2014. VaR/CVaR estimation under stochastic volatility models. International fournal of Theoretical and Applied Finance 17, 02 (2014).

[7] John C Hull. 2014. Options, futures, and other derivatives. Pearson; 9 edition.

[8] Dale W Jorgenson. 1963. Capital theory and investment behavior. The American Economic Review 53, 2 (1963), 247-259.

[9] Ladislav Kristoufek. 2013. BitCoin meets Google Trends and Wikipedia: Quantifying the relationship between phenomena of the Internet era. Scientific reports 3 (2013).

[10] Paul Malliavin, Maria Elvira Mancino, et al. 2009. A Fourier transform method for nonparametric estimation of multivariate volatility. The Annals of Statistics 37, 4 (2009), 1983-2010.

[11] Ruey S. Tsay. 2005. Analysis of Financial Time Series (2 ed.). Wiley-Interscience.

[12] Dennis van Wijk. 2013. What can be expected from the BitCoin. Erasmus Universiteit Rotterdam (2013). 